\title{
Differential activation of the default mode network in jet lagged individuals
}

\section{Joana Fernandes Coutinho, Oscar Filipe Gonçalves, Liliana Maia, Cristiana Fernandes Vasconcelos, Kristin Perrone-McGovern, Stephanie Simon-Dack, Kristina Hernandez, Patricia Oliveira-Silva, Ana Raquel Mesquita \& Adriana Sampaio}

To cite this article: Joana Fernandes Coutinho, Oscar Filipe Gonçalves, Liliana Maia, Cristiana Fernandes Vasconcelos, Kristin Perrone-McGovern, Stephanie Simon-Dack, Kristina Hernandez, Patricia Oliveira-Silva, Ana Raquel Mesquita \& Adriana Sampaio (2015) Differential activation of the default mode network in jet lagged individuals, Chronobiology International, 32:1, 143-149, DOI: 10.3109/07420528.2014.955187

To link to this article: https://doi.org/10.3109/07420528.2014.955187

Published online: 02 Sep 2014.

\section{Submit your article to this journal $[\pi$}

Шl Article views: 235

Q View related articles $\sqsubset$

View Crossmark data

Citing articles: 3 View citing articles $ऍ$ 


\title{
Differential activation of the default mode network in jet lagged individuals
}

\author{
Joana Fernandes Coutinho ${ }^{1}$, Oscar Filipe Gonçalves ${ }^{1,2,3}$, Liliana Maia ${ }^{1}$, Cristiana Fernandes \\ Vasconcelos ${ }^{4}$, Kristin Perrone-McGovern ${ }^{5}$, Stephanie Simon-Dack ${ }^{6}$, Kristina Hernandez ${ }^{6}$, Patricia \\ Oliveira-Silva $^{1}$, Ana Raquel Mesquita ${ }^{1}$, and Adriana Sampaio ${ }^{1}$ \\ ${ }^{1}$ Neuropsychophysiology Lab, CIPsi, School of Psychology, University of Minho, Braga, Portugal, ${ }^{2}$ Department of \\ Counseling and Applied Educational Psychology, Bouvé College of Health Sciences Northeastern University, Boston, \\ MA, USA, ${ }^{3}$ Spaulding Center of Neuromodulation, Spaulding Rehabilitation Hospital and Massachusetts General Hospital, \\ Harvard Medical School, Boston, MA, USA, ${ }^{4}$ Department of Neuroradiology, Hospital de Sto. António - CHP, Porto, \\ Portugal, ${ }^{5}$ Department of Counseling Psychology and Guidance Services, Ball State University, Muncie, DE, USA, and \\ ${ }^{6}$ Department of Psychological Science, Ball State University, Muncie, DE, USA
}

\begin{abstract}
Long-term exposure to transmeridian flights has been shown to impact cognitive functioning. Nevertheless, the immediate effects of jet lag in the activation of specific brain networks have not been investigated. We analyzed the impact of short-term jet lag on the activation of the default mode network (DMN). A group of individuals who were on a transmeridian flight and a control group went through a functional magnetic resonance imaging acquisition. Statistical analysis was performed to test for differences in the DMN activation between groups. Participants from the jet lag group presented decreased activation in the anterior nodes of the DMN, specifically in bilateral medial prefrontal and anterior cingulate cortex. No areas of increased activation were observed for the jet lag group. These results may be suggestive of a negative impact of jet lag on important cognitive functions such as introspection, emotional regulation and decision making in a few days after individuals arrive at their destination.
\end{abstract}

Keywords: Brain, default mode network, jet lag

\section{INTRODUCTION}

Given the impact of globalization, people travel with greater frequency and to more distant locations than they did in the past. The relevance of the biological impact of jet lag is thus justified by the increasing frequency of transmeridian flights for both professional and personal reasons.

Jet lag refers to a range of unpleasant physiological and psychological alterations experienced by individuals for few days after a transmeridian flight (i.e. traveling in a jet plane across multiple time zones) (Comperatore \& Krueger, 1990; Spitzer et al., 1999; Winget et al., 1984). These symptoms result from temporal misalignment of the endogenous circadian rhythm with external time. While the internal biological rhythm is slowly trying to adapt to new external time cues, the individuals often experience decreased alertness or fatigue (Wright et al., 1983), nighttime insomnia and other sleep disturbances including delayed sleep onset (after eastward flights), early awakening (after westward flights) and fractionated sleep (after flights in either direction (Waterhouse et al., 1997, 2007; Winget et al., 1984), poor overall performance during the new daytime at both physical and mental tasks (Graeber, 1982; Sack et al., 2007; Waterhouse et al., 1997, 2007), impaired cognitive skills (Cho et al., 2000), loss of appetite, depressed mood, irritability, reduced psychomotor coordination, gastrointestinal disturbances such as indigestion and lower frequency of defecation (Sack, 2010; Waterhouse et al., 2007) and delayed ovulation and menstrual disturbance in females (Iglesias et al., 1980).

Previous studies have shown that individuals who are repeatedly exposed to jet lag, such as business travelers and airline personnel, typically present with immune deficiencies, mood disorders, elevated cancer risk and anatomical anomalies of the forebrain (Kiessling et al.,

Submitted June 23, 2014, Returned for revision July 31, 2014, Accepted August 4, 2014

Correspondence: Joana Fernandes Coutinho, PhD, School of Psychology, University of Minho, Campus de Gualtar, 4710-057 Braga, Portugal. Tel: +351 253601398. Fax: +351 253604224. E-mail: joanafpc@gmail.com 
2010). A study by Cho et al. (2000) analyzed the effects of chronic jet lag on levels of salivary cortisol and memory function in 62 healthy women employed by international airline companies. The authors found that the cabin crew presented cognitive deficits in working memory as well as higher levels of salivary cortisol than the ground crew. Cho hypothesized, based on these results that the long-term exposure to high cortisol levels induced by flying may result in cognitive performance impairments. One year later, Cho (2001) observed a reduction in temporal lobe volume in chronically jet-lagged female flight attendants relative to controls, which was again attributed to the increased production of stress hormones. Past research has also demonstrated the biological effect of jet lag in animal models (Gibson et al., 2010; Kiessling et al., 2010; Sei et al., 2003). This research indicated that reduction in neurogenesis induced by jet lag was mediated by increased glucocorticoid concentrations.

There is a paucity of research examining the effect of jet lag on the human brain, and the few available studies like the studies by Cho et al. $(2000,2001)$ mentioned before, investigated the chronic effect of jet lag. In fact, to the best of our knowledge, there is no previous research looking at the short term effects of jet lag on brain networks known to be involved in specific psychological functions. Our study was motivated by this gap we found in the literature. We believe that knowing the particular types of cognitive deficits induced by jet lag may contribute to a better understanding of some of the jet lag symptoms mentioned before such as irritability, depressed mood and poor overall cognitive performance. We decided to study the impact of jet lag in one specific brain network - the default mode network (DMN) - due to its involvement in important psychological functions such as autobiographical memory, internal and external attention. On the other hand, the DMN has shown to be altered in anxiety and mood disorders, which have symptoms similar to some of the jet lag symptoms.

The DMN is one of the better known resting state networks and is involved in important cognitive functions. The DMN is comprised of the posterior cingulate cortex (pCC) and adjacent precuneus (PCu), the medial prefrontal cortex (mPFC), medial, lateral and inferior parietal cortex (IPC) and medial and inferior temporal cortex (ITC) (Buckner et al., 2008; Raichle et al., 2001). The DMN serves processes such as supporting internal mental activity that is detached from the external world based on personal introspection, autobiographical memories, thoughts about the future and connecting internal and external attention in monitoring the world around us (Buckner et al., 2008; Greicius et al., 2003; Mason et al., 2007). Importantly, the DMN, in particular its pattern of deactivation during the transition to goaloriented or attention-demanding tasks (Arbabshirani et al., 2013; Harrison et al., 2011), has also been related to the performance in attention demanding tasks. Thus we considered this to be a brain system that was likely to be altered when individuals are suffering from jet lag. Therefore, the main aim of this study was to analyze whether jet-lagged individuals present any alteration in the DMN functional activity on the day after their transmeridian flight, as compared to individuals in the control group.

\section{METHODS}

\section{Participants}

Twenty-four right-handed healthy volunteers composed the initial sample. The experimental group included 12 participants who were on a transmeridian flight from America to Europe (difference of seven time zones from origin to destination) 12 hours before the study. The control group included 12 participants did not fly for a period of at least one month before the study. Due to the presence of magnetic resonance image (MRI) contraindications (presence of tattoos and metallic implants) and image artifacts, four participants were excluded, thus in the final sample, each group had 10 individuals matched in age, gender (three females in each group) and educational level. Participants had a mean age of $25.6(\mathrm{SD}=5.8)$ years, ranging from 22 to 42 years. Participants were all Caucasian and held at least a Bachelor's Degree. The study was approved by the Institutional Review Board of the primary author's university. The experimental protocol applied in this study complied with international standards of ethical chronobiology research (Portaluppi et al., 2010) and the principles expressed in the Declaration of Helsinki. Exclusion criteria for this sample were as follows: (1) the presence of any major medical or neurological disorder; (2) dependency or abuse in the past year of alcohol and/or drugs; and (3) general MRI contraindications (e.g. claustrophobia, having cochlear or magnetic dental implants). The study goals and tests were explained to all participants and all gave informed written consent. After signing the written informed consent, all the participants responded to a laterality test (the Edinburgh Handedness Inventory, Oldfield, 1971).

\section{Data acquisition}

A five-minute resting-state functional MRI scan was acquired using a clinically approved Philips Achieva 3T (Centro Hospitalar do Porto, Portugal) equipped with an eight-channel head coil. Functional images were obtained axially using a gradient-echo echo-planar imaging (EPI) BOLD fMRI acquisition sequence with the following imaging parameters: repetition time $=3 \mathrm{~s}$, echo time $=40 \mathrm{~ms}$, flip angle $=90^{\circ}$, field of view $=235$ $\mathrm{mm}$, slice thickness $=3.2 \mathrm{~mm}, 45$ axial slices with a matrix $=72 \times 74 ; \quad$ voxel $\quad$ size $=2.9 \times 2.9 \times 3.2 \mathrm{~mm}^{3}$. Participants were instructed to keep their eyes closed and to remain awake but relaxed and doing nothing in particular during the acquisition. This was the instruction given to the participants because in studies that 
analyze the brain's resting activity (contrary to classical fMRI studies), there is no specific task that the subject needs to perform. In other words, in these studies, we measure the brain's resting activity (i.e. the BOLD signal change during the five minutes of scanning) as a whole. Therefore, all the areas of the DMN are included in the analysis in order to explore whether there were any differences between both groups in terms of DMN activity.

\section{Image pre-processing}

The preprocessing of the fMRI data was performed using Data Processing Assistant for Resting-State fMRI (Chao-Gan \& Yu-Feng, 2010). The first six volumes from each subject were discarded to allow the signal to reach equilibrium and to allow the participants to adapt to the scanning noise. The remaining 94 volumes in the functional scan were included in the analysis. Head motion parameters were then estimated, and each volume was realigned to the mean map of the whole volumes to correct for geometric displacements using a six-parameter rigid-body transformation. All subjects displayed head motion less than $1.5 \mathrm{~mm}$ in translation or $2^{\circ}$ in rotation.

The data were first corrected for slice timing using the midline slice before being normalized into Montreal Neurological Institute space using the default EPI templates in the toolbox and resampled to $3 \mathrm{~mm} \times 3 \mathrm{~mm} \times 3 \mathrm{~mm}$. Detrending and filtering with a default value of $0.01-0.08 \mathrm{~Hz}$ was performed on the time series of each voxel to reduce the effects of lowfrequency drift and high-frequency noise. Images were then smoothed with 8-mm Full-Width, Half-Maximum.

Spatial ICA was performed using the GIFT software v1.3g (http://icatb.sourceforge.net) using the IFOMAX algorithm (Bell \& Sejnowski, 1995; Calhoun et al., 2001). The ICASSO tool was used to control the ICA reliability. On the process, 15 independent sources were estimated on the aggregate data. Individual subject ICA data sets were then back-reconstructed. The DMN component was identified visually and by selecting the component with the highest spatial correlation to the default network template supplied by the GIFT software.

\section{Statistical analyses}

Our main statistical analysis compared the DMN activation (global activation in all the areas that constitute this brain network) in the jet-lag group versus the control group.

The statistical analyses using the second level random effect was performed on SPM8 (Statistical Parametrical Mapping, version 8, http://www.fil.ion.ac.uk) in two steps. First, we used a one sample $t$-test to confirm the DMN activation in both groups. It is common to present the map of activation of the DMN for the entire sample to make sure that all the participants presented an activation on the brain network under study. Otherwise, some individuals who could not show a coherent pattern of activation of the DMN (due to any neurological disorder for example) could bias the statistical analysis of the difference between groups.

Then, a directional two sample $t$-test was used for between groups comparison. All the results were corrected for multiple comparisons applying false discovery rate (FDR) correction (Kucian et al., 2006). In the neuroimaging field, it is likely that we find differences of activation that constitute false positives, due to the number of statistical tests performed. Thus researchers address this issue by using procedures of correction for multiple comparisons. FDR is a commonly used procedure of correction for multiple comparisons that aims to prevent type I statistical errors.

The results presented were masked using the default network template supplied by the GIFT software, the same used on pre-processing.

\section{RESULTS}

\section{DMN global activation}

The DMN was identified in the resting state conditions at the group level, and four main components were observed, namely the pCC and PCu; the MPFC; the bilateral IPC; and the left ITC (see Figure 1).

\section{Group differences in DMN activation}

We found that participants from the jet lag group, when compared with the control group, presented decreased activation in several DMN regions: bilateral medial frontal gyrus $(x=-6, y=51, z=-3)$; right anterior cingulate $(x=6, y=42, z=0)$; left parahippocampal gyrus ( $x=-24, y=-33, z=-12$ ) and left angular gyrus $(x=-51, y=-69, z=30)$ (see Table 1 and Figure 2).

As is typically done in neuroimaging studies, for each cluster (a set of voxels that form a brain region), we report the peak voxel, that is, the unit of volume that corresponds to the voxel in which the activation was higher, and its size (the number of voxels that belong to that same area).

These results were significant at a corrected for multiple comparisons $p<0.05$ threshold (FDR) and a minimum cluster size of 20 voxels. We only reported clusters that had a minimum of 20 voxels because very small clusters normally do not represent a meaningful activation, so we determine a minimum size for a cluster to be considered significant.

\section{DISCUSSION}

In this study, we aimed to explore the impact of the acute circadian disruption caused by jet lag on the brain activity at rest, namely on the patterns of activation of the DMN. We found that after a transmeridian flight, individuals presented lower functional activity in several areas of this resting state brain network. Specifically, our findings show that jet-lagged individuals presented a 
FIGURE 1. DMN activation identified in the resting state condition at the group level ( $p<0.05$ FWE corrected for multiple comparisons). In this picture that corresponds to the map of activation of the DMN for the entire sample, we can see a sagittal section showing the medial prefrontal cortex and the posterior cingulate cortex (left image on the top); a coronal section showing the posterior cingulate cortex (right image on the top) and an axial section (bellow) showing the medial prefrontal cortex, posterior cingulate cortex and lateral parietal cortex. The scale represents the degree of activation, in other words, the lighter a region is, the stronger was the activation in that brain region.
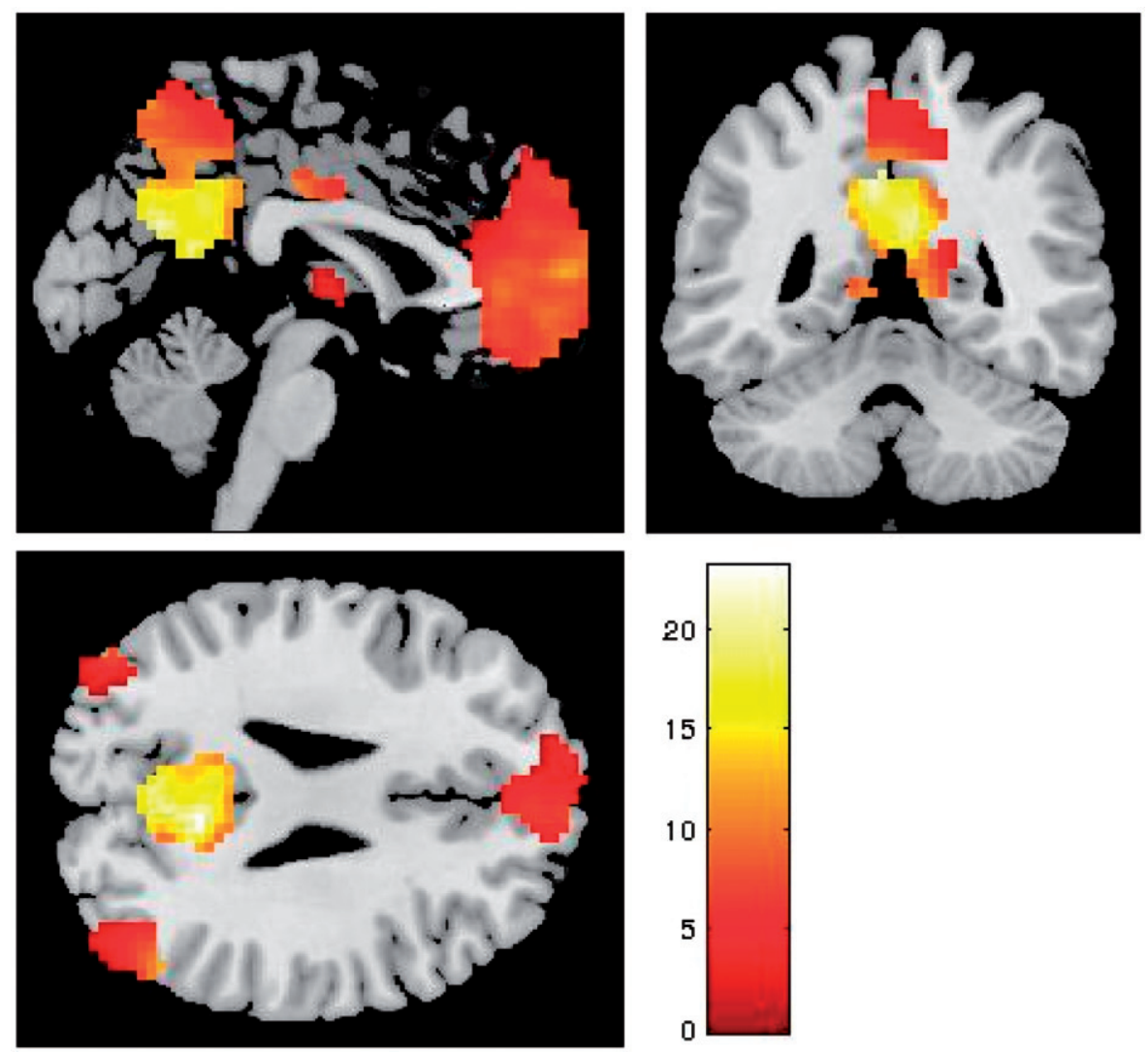

TABLE 1. DMN areas that presented a decreased activation in the jet lag group.

\begin{tabular}{lccccc}
\hline Brain region & $T$ value & Z value & $p$ & $\mathrm{~K}$ (cluster size) & MNI coordinates $(x, y, z)$ \\
\hline Left medial frontal gyrus (mPFC) & 4.21 & 3.47 & 0.013 & \multirow{2}{*}{130} & $-6,51,-3$ \\
Right anterior cingulate gyrus (ACC) & 4.09 & 3.39 & 0.014 & & $6,42,0$ \\
Right medial superior frontal gyrus & 5.04 & 3.93 & 0.006 & 263 & $9,57,33$ \\
Left middle frontal gyrus & 4.94 & 3.88 & 0.007 & & $-24,27,51$ \\
Left parahippocampal gyrus & 6.02 & 4.4 & 0.003 & 21 & $-24,-33,-12$ \\
Left angular gyrus & 4.6 & 3.69 & 0.008 & 23 & $-51,-69,30$ \\
\hline
\end{tabular}

Each cluster's coordinates in Montreal Neurological Institute (MNI) space $(x, y, z)$, maximum T and $\mathrm{Z}$ value and size are given. All results were significant at a corrected for multiple comparisons $p<0.05$ threshold (FDR) and a minimum cluster size of 20 voxels.

lower resting activation in two anterior regions of the DMN: the anterior cingulate cortex and mPFC. These brain regions are known to be involved in crucial psychological processes such as decision making, selective attention and regulation of emotional responses (e.g. Buckner et al., 2008; Lane et al., 1997). Therefore, the decreased activity in these DMN areas is consistent with the impaired psychological functioning that is frequently reported by people suffering from jet lag (Sack, 2010; Sack et al., 2007).

Our findings suggest that the immediate effects of jet lag in the brain may compromise the performance in cognitive functions that are often required to individuals after arriving at their destination, in business commitments or professional affairs. These results are consistent with Wright et al. (1983) findings, which demonstrated that rapid time-zone transitions are associated with cognitive and physical performance impairments, with some of the subjects being unable to even complete the tasks. Similarly, the results by Cho et al. (2000) suggested that chronic jet lag may depress nonverbal short-term memory processing.

Therefore, the results from this study make a significant contribution to existing literature on how the disturbance of circadian rhythms affects individuals' performance on a short-term basis, by showing the presence of altered brain resting state patterns. In fact, the anterior nodes of the DMN such as the mPFC play a central role in the regulation of emotional arousal (e.g. Etkin et al., 2006; Tillfors et al., 2002; Zubieta et al., 1999), whereas the adjacent ACC has been shown to be involved in affective processing of negative information (Amir et al., 2005), and to be especially sensitive to internal signs of autonomic arousal (Shin et al., 2013). The fact that these areas were less active suggests that jet lagged individuals may have more 

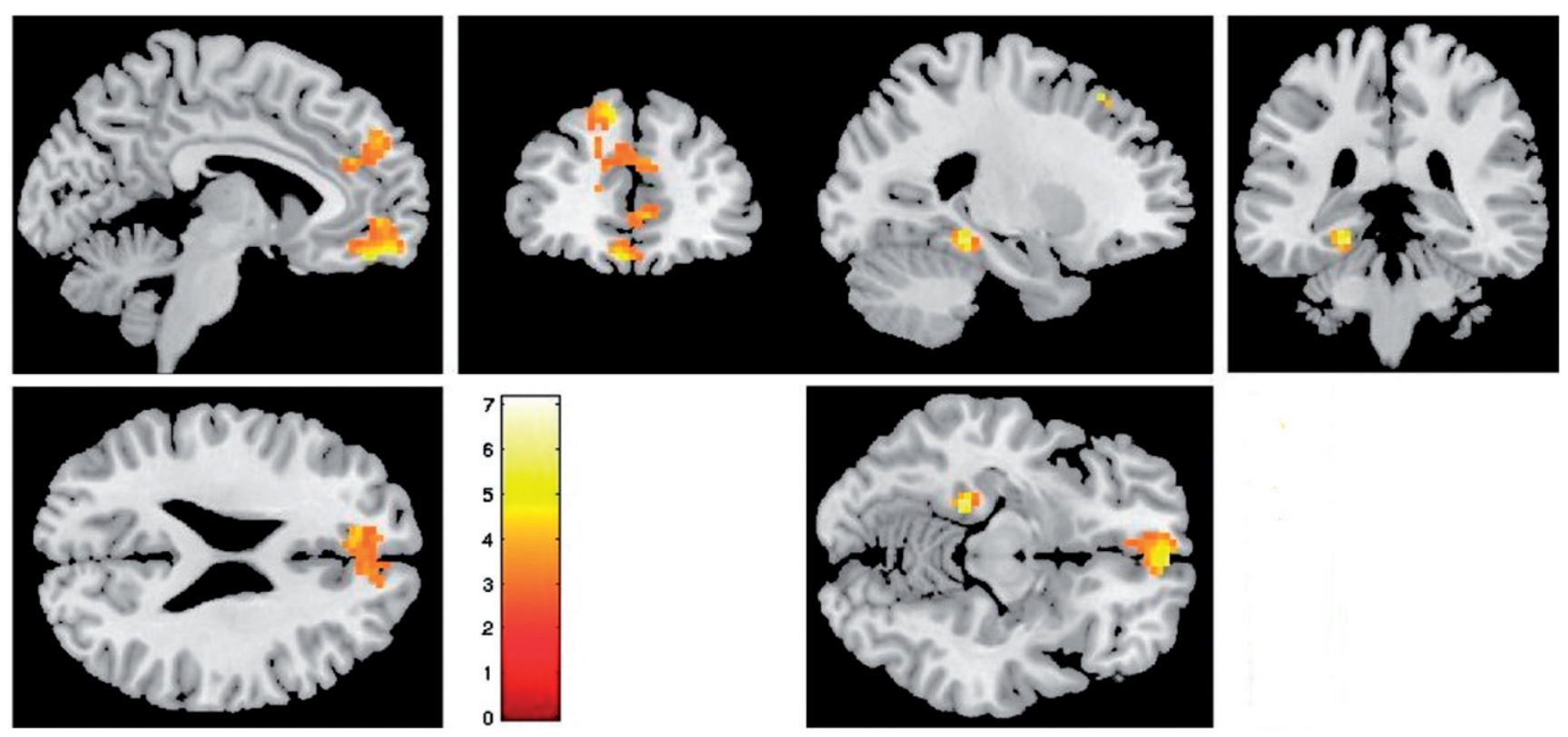

FIGURE 2. DMN regions with decreased activation in the jet lag group ( $p<0.05$ FDR-corrected for multiple comparisons). The images show sagittal, coronal and axial sections of the brain regions with decreased activation in the jet lag group: medial prefrontal and anterior cingulate cortex (image on the left) and the parahippocampal regions (right image). The scale indicates the magnitude of the difference between groups: the lighter a region is, the less active it was in jet lag in comparison with the control group.

difficulty dealing with stressful events, which in turn is consistent with previous data reporting higher levels of cortisol during jet lag, and with the fact that symptoms reported by jet lagged individuals often include loss of mental efficiency, weakness and irritability (Comperatore \& Krueger, 1990).

We also found that the left parahippocampal and angular brain regions were less active in jet lagged individuals. These nodes of the DMN are associated with autobiographical memory (Olson et al., 2007; Spreng \& Grady, 2010; Spreng \& Mar, 2012) and visual and sensory processing (Ganis et al., 2004). Our results showing that these memory nodes of the DMN presented a decreased activation in jet lagged individuals are in line with previous studies that found evidence of memory deficits in jet lag. However, it is important to note that those studies (Cho et al., 2000; Lupien et al., 1998) attributed the memory impairment to the longterm effects of jet-lag and associated repeated exposure to high cortisol levels. Indeed, several studies demonstrated that high levels of circulating corticosteroids induced decreased memory performance (McEwen \& Sapolsky, 1995; Newcomer et al., 1999) and impaired hippocampal-dependent learning (Lupien et al., 1994, 1998). The inclusion of a measure of cortisol will thus be important for future studies.

We cannot establish a real causal link between jet lag and the alterations in the DMN activation, because we cannot exclude other factors that may also affect brain activity at rest. One potential confound in this study is sleep deprivation that is a typical concomitant symptom of jet lag but may itself be a factor that modulates attention and cognitive performance. It has been shown that sleeplessness disrupts neurogenesis in both a glucocorticoid-dependent and independent manner (Mirescu et al., 2006; Mueller et al., 2008). However, previous studies have shown that short-term sleep deprivation (36 hours) does not selectively impair higher cortical functioning (Binks et al., 1999). Likewise, studies using animal models (Gibson et al., 2010) suggest that it is unlikely that the effects of jet lag on hippocampal structure and function are mediated by disruptions in the activity/rest cycle, as in these studies the total duration of the active and inactive phases of the circadian cycle were not different between the control and the jet lag group.

In addition, it is not easy to separate the biological effects of circadian rhythm alteration from the stress caused by the adaptation to the new context, the trip preparation, etc.

Future functional neuroimaging studies are needed to determine whether the alteration in the DMN activity that we found in this study persists for some days after the flight. Moreover, future studies should explore the effect of different types of circadian desynchrony by considering both phase advances and phase delays in circadian rhythms. In the same line, it is important to look at the effects of rotating shift work schedules, night work, etc., which like transmeridian travel also results in an incongruence between the endogenous circadian timing system and the external environment. Finally, we believe that future studies should include a self-report measure of jet lag (Spitzer et al., 1999), in order to analyze whether the brain alterations found in our study increase with the severity of jet lag symptoms. 
The frequency of long-distance flights motivated by professional and personal issues is increasing significantly. More than 30 million travelers in the United States alone are affected (Sack, 2010). International companies and individuals who travel frequently could benefit from a better understanding of how brain functioning is immediately affected by jet lag.

\section{DECLARATION OF INTEREST}

The authors have no conflicts of interest to declare.

This study was funded by the Portuguese Foundation for Science and Technology (FCT): PIC/IC/83290/2007, which is supported by FEDER (POFC - COMPETE). Joana Coutinho was funded by a FCT postdoctoral grant (number: SFRH/BPD/75014/2010) and Bial Foundation (grant number 87/12). Liliana Maia is supported by the Competitive Factors Operational Programme COMPETE - by national funds from the Portuguese Foundation for Science and Technology (grant PTDC/ PSI-PCL/115316/2009).

\section{REFERENCES}

Amir N, Klumpp H, Elias J, et al. (2005). Increased activation of the anterior cingulate cortex during processing of disgust faces in individuals with social phobia. Biol Psychiatry. 57:975-81.

Arbabshirani MR, Havlicek M, Kiehl KA, et al. (2013). Functional network connectivity during rest and task conditions: A comparative study. Hum Brain Map. 34:2959-71.

Bell AJ, Sejnowski TJ. (1995). An information-maximization approach to blind separation and blind deconvolution. Neural Comput. 7:1129-59.

Binks PG, Waters WF, Hurry M. (1999). Short-term total sleep deprivations does not selectively impair higher cortical functioning. Sleep. 22:328-34.

Buckner RL, Andrews-Hanna JR, Schacter DL. (2008). The brain's default network. Ann NY Acad Sci. 1124:1-38.

Calhoun VD, Adali T, Pearlson GD, Pekar JJ. (2001). A method for making group inferences from functional MRI data using independent component analysis. Hum Brain Map. 14:140-51.

Chao-Gan Y, Yu-Feng Z. (2010). DPARSF: A MATLAB toolbox for "pipeline" data analysis of resting-state fMRI. Front Syst Neurosci. 4:13. doi: 10.3389/fnsys.2010.00013.

Cho K, Ennaceur A, Cole JC, Suh CK. (2000). Chronic jet lag produces cognitive deficits. J Neurosci. 20:RC66 (1-5).

Cho K. (2001). Chronic 'jet lag' produces temporal lobe atrophy and spatial cognitive deficits. Nat Neurosci. 4:567-8.

Comperatore CA, Krueger GP. (1990). Circadian rhythm desynchronosis, jet lag, shift lag and coping strategies. Occup Med. 5: 323-42.

Etkin A, Egner T, Peraza DM, et al. (2006). Resolving emotional conflict: A role for the rostral anterior cingulate cortex in modulating activity in the amygdala. Neuron. 51:871-82.

Ganis G, Thompson WL, Kosslyn SM. (2004). Brain areas underlying visual mental imagery and visual perception: An fMRI study. Cogn Brain Res. 20:226-41.

Gibson EM, Wang C, Tjho S, et al. (2010). Experimental 'jet lag'inhibits adult neurogenesis and produces long-term cognitive deficits in female hamsters. PLoS One. 5:e15267.

Graeber RC. (1982). Alterations in performance following rapid transmeridian flight. In Brown FM, Graeber RC, eds. Rhythmic aspects of behavior. Hillsdale, NJ: Lawrence Erlbaum Associates, pp. 173-212.

Greicius MD, Krasnow B, Reiss AL, Menon V. (2003). Functional connectivity in the resting brain: A network analysis of the default mode hypothesis. PNAS. 100:253-8.

Harrison BJ, Pujol J, Contreras-Rodríguez O, et al. (2011). Task-induced deactivation from rest extends beyond the default mode brain network. PLoS One. 6:e22964.

Iglesias R, Terres A, Chavarria A. (1980). Disorder of the menstrual cycle in airline stewardesses. Aviat Space Environ Med. 51: 518-20.

Kiessling S, Eichele G, Oster H. (2010). Adrenal glucocorticoids have a key role in circadian resynchronization in a mouse model of jet lag. J Clin Invest. 120:2600-9.

Kucian K, Loenneker T, Dietrich T, et al. (2006). Impaired neural networks for approximate calculation in dyscalculic children: A functional MRI study. Behav Brain Func. 31:1-17.

Lane RD, Reiman B, Axelrod, et al. (1997). Neural correlates of levels of emotional awareness: Evidence of an interaction between emotion and attention in the anterior cingulate cortex. J Cogn Neurosci. 10:525-35.

Lupien S, Lecours AR, Luster I, et al. (1994). Basal cortisol levels and cognitive deficits in human aging. J Neurosci. 14: 2893-903.

Lupien SJ, de Leon M, de Santi S, et al. (1998). Cortisol levels during human aging predict hippocampal atrophy and memory deficits. Nat Neurosci. 1:69-73.

Mason ML, Norton MI, Van Horn JD, et al. (2007). Wandering minds: The default network and stimulus-independent thought. Science. 215:393-5.

McEwen BS, Sapolsky RM. (1995). Stress and cognitive function. Cur Opin Neurobiol. 5:205-16.

Mirescu C, Peters JD, Noiman L, Gould E. (2006). Sleep deprivation inhibits adult neurogenesis in the hippocampus by elevating glucocorticoids. PNAS. 103:19170-5.

Mueller AD, Pollock MS, Lieblich SE, et al. (2008). Sleep deprivation can inhibit adult hippocampal neurogenesis independent of adrenal stress hormones. Am J Physiol Regul Integr Comp Physiol. 294:1693-703.

Newcomer JW, Selke G, Melson AK, et al. (1999). Decreased memory performance in healthy humans induced by stresslevel cortisol treatment. Arch Gen Psychiatry. 56:527-33.

Olson IR, Plotzker A, Ezzyat Y. (2007). The enigmatic temporal pole: A review of findings on social and emotional processing. Brain. 130:1718-31.

Portaluppi F, Smolensky MH, Touitou Y. (2010). Ethics and methods for biological rhythm research on animals and human beings. Chronobiol Int. 27:1911-29.

Raichle ME, MacLeod AM, Snyder AZ, et al. (2001). A default mode of brain function. PNAS. 98:676-82.

Sack RL, Auckley D, Auger RR, et al. (2007). Circadian rhythm sleep disorders: Part I, basic principles,shift work and jet lag disorders. Sleep. 30:1460-83.

Sack RL. (2010). Jet lag. N Engl J Med. 362:440-7.

Sei H, Fujihara H, Ueta Y, et al. (2003). Single eight-hour shift of light-dark cycle increases brain-derived neurotrophic factor protein levels in the rat hippocampus. Life Sci. 73:53-9.

Shin YW, Dzemidzic M, Jo HJ, et al. (2013). Increased resting-state functional connectivity between the anterior cingulate cortex and the precuneus in panic disorder: Resting-state connectivity in panic disorder. J Affect Disord. 150:1091-5.

Spitzer RL, Terman M, Williams JB, et al. (1999). Jet lag: Clinical features, validation of a new syndrome-specific scale, and lack of response to melatonin in a randomized, double-blind trial. Am J Psychiatry. 156:1392-6.

Spreng RN, Grady CL. (2010). Patterns of brain activity supporting autobiographical memory, prospection, and theory of mind, and their relationship to the default mode network. J Cogn Neurosci. 22:1112-23. 
Spreng RN, Mar RA. (2012). I remember you: A role for memory in social cognition and the functional neuroanatomy of their interaction. Brain Res. 1428:43-50.

Tillfors M, Furmark T, Marteinsdottir I, Fredrikson M. (2002). Cerebral blood flow during anticipation of public speaking in social phobia: A PET study. Biol Psych 52:1113-19.

Waterhouse J, Atkinson G, Reilly T. (1997) Jet lag. Lancet. 350: 1611-16.

Waterhouse J, Reilly T, Atkinson G, Edwards B. (2007). Jet lag: Trends and coping strategies. Lancet. 369:1117-29.
Winget CM, DeRoshia CW, Markley CL, Holley DC. (1984). A review of human physiological and performance changes associated with desynchronosis of biological rhythms. Aviat Space Environ Med. 55:1085-96.

Wright JE, Vogel JA, Sampson JB, et al. (1983). Effects of travel across time zone (jet-lag) on exercise capacity and performance. Aviat Space Environ Med. 54:132-7.

Zubieta JK, Chinitz JA, Lombardi U, et al. (1999). Medial frontal cortex involvement in PTSD symptoms: A SPECT study. J Psychiatr Res. 33:259-64. 